\title{
Parity Violation in Deep Inelastic Scattering with the SoLID Spectrometer at JLab
}

\author{
P. A. Souder \\ Department of Physics, 201 Physics Building, Syracuse University \\ Syracuse, New York, 13244, USA \\ souder@phy.syr.edu
}

Published 29 February 2016

\begin{abstract}
We discuss precision measurements of PVDIS with the upgraded JLab $12 \mathrm{GeV}$ beam by using a Solenoidal Large Iintensity Device (SoLID). The unique feature of SoLID, combining high luminosity and large acceptance, makes it possible to reach the high precision needed to have a high impact by using PVDIS to probe physics beyond the Standard Model. A measurement of PVDIS in deuterium will determine a fundamental coupling constant that is inaccessible with other means. PVDIS measurements can also access a number of topics in QCD physics, including searching for charge symmetry violation in the parton distribution functions, determining the $d / u$ ratio in the proton without nuclear effects, and a clean extraction of higher-twist effects due to quark-quark correlations. SoLID allows a full exploitation of the physics potential of the JLab $12 \mathrm{GeV}$ upgrade. In addition to PVDIS, it has a set of approved highly-rated experiments to study nucleon transverse spin and transverse structure by using polarized semi-inclusive DIS and to study non-perturbative gluon dynamics with $\mathrm{J} / \Psi$ production near threshold. A brief description of the SoLID spectrometer is also given.
\end{abstract}

Keywords: Parity-violation, deep inelastic scattering.

PACS numbers: $24.80+\mathrm{y} 24.85+\mathrm{p} 11.30 \mathrm{Er} 13.60 \mathrm{Hb}$

\section{Introduction}

In the late 70's, Prescott et al. ${ }^{1,2}$ showed, by measuring the non-zero asymmetry $A_{L R}=\left(\sigma_{L}-\sigma_{R}\right) /\left(\sigma_{L}+\sigma_{R}\right)$ for polarized electron-deuterium deep inelastic scattering, that the weak neutral current violates parity . The experiment also set limits on the dependence of $A_{L R}$ on the variable $y \equiv\left(E-E^{\prime}\right) / E$, which ruled out models invented to explain the negative results of the early atom parity violation (APV) experiments. Subsequent to these publications, Glashow, Salam, and Weinberg were awarded the Nobel prize for electroweak unification.

This is an Open Access article published by World Scientific Publishing Company. It is distributed under the terms of the Creative Commons Attribution 3.0 (CC-BY) License. Further distribution of this work is permitted, provided the original work is properly cited. 

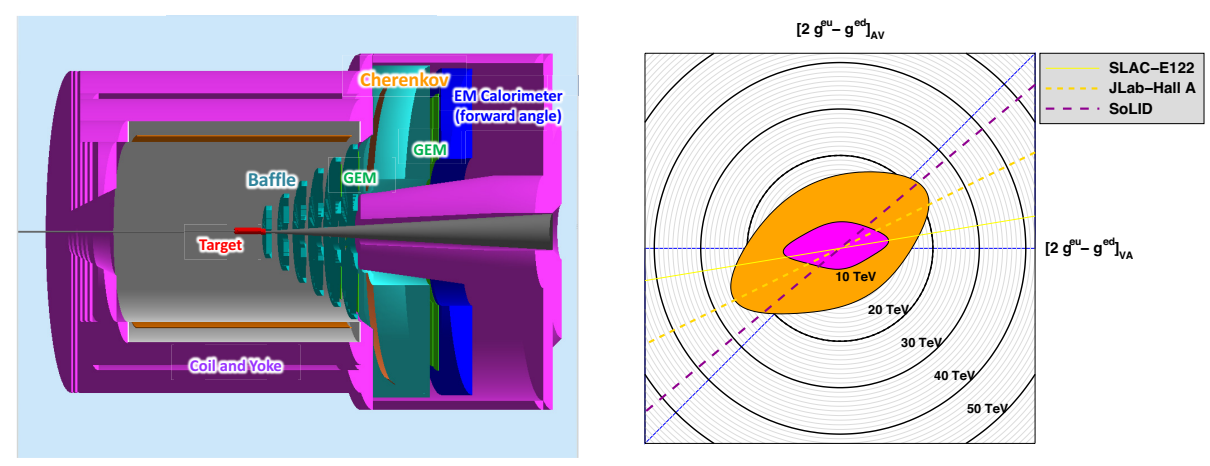

Fig. 1. Left panel: SoLID apparatus. Scattered electrons spiral through the baffles and are detected by GEM's and identified with a Cerenkov counter and an EM calorimeter. Right panel: Projected mass limits for composite models. Purple region is excluded by published data and the orange region is the projected result with SoLID and final Qweak result.

Parity violating electron scattering (PVES) from deuterium in the DIS region at large Bjorken $x$ is an attractive reaction for searching for new physics since there the $A_{L R}$ is approximately independent of hadron structure. The remaining QCD effects, which are of great interest in themselves, can be isolated by their kinematic dependence. The asymmetry has the form ${ }^{3} A / Q^{2}=-\left[a_{1}+a_{3} f(y)\right]$, where $f(y) \approx\left[1-(1-y)^{2}\right] /\left[1+(1-y)^{2}\right]$ and $a_{1} \propto 2 C_{1 u}-C_{1 d}$ and $a_{3} \propto 2 C_{2 u}-C_{2 d}$, where $C_{1 q}\left(C_{2 q}\right)$ are four-Fermi coupling constants with axial(vector) electron currents and vector(axial) quark currents. At large $y, A_{L R}$ is sensitive to the $C_{2 q}$, which cannot be studied in low energy reactions because of large and uncertain radiative corrections. Experiments with atomic parity violation ${ }^{4,5}$ and PVES experiments including Qweak ${ }^{6}$ have yielded precise measurements of the $C_{1 q}$. The $6-\mathrm{GeV}$ PVDIS collaboration ${ }^{7}$ has recently published in Nature a new experimental result $2 C_{2 u}-C_{2 d}=-0.145 \pm 0.068$, the first measurement sufficiently sensitive to show that the $C_{2 q}$ are non-zero as predicted by the SM.

It is important to improve the measurement of the $C_{2 q}$ to the level of the $C_{1 q}$. This requires a facility with both high luminosity and large acceptance which leads to the idea of SoLID. ${ }^{8-10}$ We have developed a preliminary conceptual design of a spectrometer, SoLID (left panel of Fig. 1), to reach this goal. ${ }^{11}$ The apparatus can measure $A_{P V}$ for about 20 kinematic points with $x>0.4$ and a range of $Q^{2}$ each with a statistical precision of about $0.5 \%$.

\section{Physics Reach}

One way to quantify the reach of various experiments is to quote mass limits suitable for composite models, ${ }^{12}$ where the couplings are on the order of $4 \pi / \Lambda^{2}$ with $\Lambda$ is the compositeness mass scale. Such limits for the 6-GeV PVDIS collaboration and the SoLID PVDIS experiment ${ }^{13}$ are shown in the right panel of Fig. 1. The mass limits are on the scale probed by the LHC, but have the unique feature that they 
have a very specific helicity structure. For example, a lepto-phobic $Z^{\prime 14}$ can only contributes to the $C_{2 q}$. In addition, a broken $\mathrm{U}(1)$ gauge symmetry in the dark matter sector can induce a change in $\sin ^{2} \theta_{W}$ that can be observed in low $Q^{2}$ PVES experiments. ${ }^{15}$

Uncertainties in hadron structure, including charge symmetry violation (CSV) in the PDF's ${ }^{16}$ and $Q^{2}$-dependence due to higher twist effects, ${ }^{17}$ which would be of concern for a single kinematic point. With a wide kinematic range in both $x$ and $Q^{2}$, we can separate out these effects. We note that if these hadronic effects are large enough to be observed by SoLID, they will constitute important discoveries in and of themselves. Further, by using a ${ }^{48} \mathrm{C}$ target, the SoLID spectrometer can observe a possible isovector $\mathrm{EMC}^{18}$ effect that is predicted to be large enough ${ }^{19}$ to explain the $\mathrm{NuTeV}$ anomaly. ${ }^{20}$

\section{Measurement of the PDF Ratio $d / u$ Independent of Nuclear Structure}

A measurement of $A_{L R}^{\mathrm{DIS}}$ on a proton target is sensitive ${ }^{8}$ to the ratio of the $d$ to $u$ quark PDF's. The standard determination of the $d / u$ ratio relies on fully inclusive DIS cross section on a proton target compared to that for a deuteron target. In the large $x$ region, nuclear corrections in the deuteron target lead to large uncertainties in the $d / u$ ratio. However, they can be completely eliminated if the $d / u$ ratio is obtained from the proton target alone. For this reason, precision measurements of $A_{L R}^{\mathrm{DIS}}$ on a proton target can be a powerful probe of the $d / u$ ratio.

$A_{L R}$ for a proton target at leading twist takes the form,

$$
A_{L R}^{p} \sim-\frac{1}{4 \pi \alpha} \frac{Q^{2}}{v^{2}}\left[\frac{12 g_{A V}^{e u}-6 g_{A V}^{e d} d / u}{4+d / u}\right],
$$

As in the case of the deuteron asymmetry, other hadronic corrections can affect the extraction of the $d / u$ ratio. The data will be complementary to proposed experiments at JLab including one using mirror ${ }^{3} \mathrm{H}$ and ${ }^{3} \mathrm{He}$ nuclei to minimize nuclear effects in extracting $d / u,{ }^{21}$ and the BONUS experiment ${ }^{22}$ by tagging slow moving proton to minimize nuclear effects in extracting the ratio.

\section{The Full SoLID Program}

The SoLID spectrometer is also designed to provide a rich program in Semi-Inclusive Deep Inelastic Scattering (SIDIS) to measure Transverse Momentum Dependent Parton Distributions (TMD's) and also $J / \Psi$ production. The unique feature of combining large acceptance and high luminosity of the SoLID makes it possible to exploit the full potential of the JLab $12 \mathrm{GeV}$ to perform precision study of the nucleon structure structure and QCD dynamics. A set of SIDIS experiments will map the transverse structure of the nucleon with unprecedented precision. Threshold $\mathrm{J} / \Psi$ production will provide important information on non-perturbative gluon dynamics. 


\section{Overview of SoLID Instrumentation}

The base equipment composing the SoLID project includes two configurations: the "SIDIS\&J/ $\psi$ " configuration and the "PVDIS" configuration. Although the geometrical layouts for the detectors are not the same in the two configurations, most components of the following list of the SoLID base equipment are in common:

- A solenoidal magnet with a power supply and cryogenic system, now identified as the CLEO-II magnet. With some modifications as described in the pCDR, ${ }^{11}$ this magnet meets the experimental requirements.

- GEM detectors for tracking: These are planned to be provided by a SoLID Chinese Collaboration. Five Chinese institutions (USTC, CIAE, Tsinghua, Lanzhou and IMP), in collaboration with US groups (UVa and Temple), have committed to perform R\&D and plan to apply for funding from the Chinese funding agencies to construct the GEMs for the SoLID project.

- An electromagnetic calorimeter for electron identification. (In the SIDIS configuration, it is separated into two sectors, a forward sector and a large-angle sector).

- A light gas Cherenkov detector for electron identification.

- A heavy gas Cherenkov detector for pion (hadron) identification. This is for the SIDIS configuration only.

- A MRPC (Multi-Gap Resistive Plate Chamber) detector serving as a time-offlight (TOF) detector for pion (hadron) identification. This is for the SIDIS configuration only. The Chinese groups (Tsinghua and USTC), in collaboration with US groups (Duke and Rutgers), have agreed to perform R\&D and apply for funding to construct the required MRPC detector for the SoLID project.

- A set of baffles to reduce background. This is for the PVDIS configuration only.

- A data acquisition system (DAQ) with online farm capability.

\section{Summary}

The SoLID spectrometer has the capability to improve the precision of the measurement of the $C_{2 i}$ coupling constants by almost an order of magnitude to provide a new window on possible physics beyond the SM and also search for some important hadronic effects. The SoLID apparatus, which features both large acceptance and high luminosity, will be used for an extensive physics program, including SIDIS and $J \Psi$ production. The project will undergo a JLab directors review in Feb. 2015. We are hoping to start the project in FY 1918.

\section{Acknowledgments}

This material is based upon work supported in part by US Department of Energy, Office of Science, Office of Nuclear Physics under contract number DE-FG0284ER40146. 


\section{References}

1. C. Y. Prescott et al., Phys. Lett. B 77, 347 (1978)

2. C. Y. Prescott et al., Phys. Lett. B 84, 524 (1979).

3. T. Hobbs and W. Melnitchouk, Phys. Rev. D 77, 114023 (2008).

4. C. Wood et al., Science 275, 1759 (1997).

5. S. Porsev, K. Beloy, and A. Derevianko, Phys.Rev. D82, 036008 (2010), 1006.4193.

6. D. Androic et al. [Qweak Collaboration], Phys. Rev. Lett. 111, no. 14, 141803 (2013)

7. D. Wang et al. [PVDIS Collaboration], Nature 506, no. 7486, 67 (2014).

8. P. A. Souder, AIP Conf. Proc. 747, 199 (2005).

9. P. A. Souder, Parity-violating DIS at $12 \mathrm{GeV}$, in From Parity Violation to Hadronic Structure and more..., eds K. De Jager, et al., (Springer, Berlin, 2007), p. 211.

10. Jefferson Lab Proposals PR-09-012 and PR-10-007, P. A. Souder, contact person.

11. SoLID Collaboration, "Solid (solenoidal large intensity device) preliminary conceptual design report, 2014", http://hallaweb.jlab.org/12GeV/SoLID/download/doc /solid_precdr.pdf.

12. E. Eichten, K. D. Lane and M. E. Peskin, Phys. Rev. Lett. 50, 811 (1983).

13. J. Erler, C. J. Horowitz, S. Mantry and P. A. Souder, Annual Review of Nuclear and Particle Science, Volume 64, 2014

14. M. Gonzlez-Alonso and M. J. Ramsey-Musolf, Phys. Rev. D 87, no. 5, 055013 (2013)

15. H. Davoudiasl, H. S. Lee and W. J. Marciano, Phys. Rev. D 89, no. 9, 095006 (2014)

16. J. T. Londergan and A. W. Thomas, Phys. Lett. B 558, 132 (2003).

17. S. Mantry, M. J. Ramsey-Musolf and G. F. Sacco, Phys. Rev. C 82, 065205 (2010).

18. I. C. Cloet, W. Bentz and A. W. Thomas, Phys. Rev. Lett. 109, 182301 (2012)

19. W. Bentz, I. C. Cloet, J. T. Londergan and A. W. Thomas, Phys. Lett. B 693, 462 (2010).

20. G. P. Zeller et al. [NuTeV Collaboration], Phys. Rev. Lett. 88, 091802 (2002) [Erratum-ibid. 90, 239902 (2003)].

21. J. Arrington, J. G. Rubin and W. Melnitchouk, Phys. Rev. Lett. 108, 252001 (2012)

22. N. Baillie et al. [CLAS Collaboration], Phys. Rev. Lett. 108, 142001 (2012) [Erratumibid. 108, 199902 (2012)] 